\title{
Theory of ac Stark splitting in core-resonant Auger decay in strong x-ray fields
}

\author{
L. A. A. Nikolopoulos, ${ }^{*}$ T. J. Kelly, and J. T. Costello \\ School of Physical Sciences, Dublin City University and NCPTL, Dublin 9, Ireland \\ (Received 13 September 2011; revised manuscript received 6 November 2011; published 21 December 2011)

\begin{abstract}
In this work we report the modification of the normal Auger line shape under the action of an intense x-ray radiation. Under strong Rabi-type coupling of the core, the Auger line profile develops into a doublet structure with an energy separation mainly determined by the relative strength of the Rabi coupling. In addition, we find that the charge resolved ion yields can be controlled by judicious choice of the x-ray frequency.
\end{abstract}

DOI: 10.1103/PhysRevA.84.063419

PACS number(s): 32.80.Hd, 33.20.Xx, 41.60.Cr

\section{INTRODUCTION}

The interaction of an atomic system with a radiation field in the regime of $\mathrm{x}$ rays will lead to its ionization. The most dominant process will be, first, the ejection of an inner-shell electron (photoelectron) with the absorption of a photon followed either by an intra-atomic Auger and/or a fluorescence transition. For relatively light atomic systems, the dominant decay channel of the single-hole singly charged system is through an (radiationless) Auger transition, designated as "normal Auger" which is a manifestation of electron-electron interaction. One variation on this scenario is to promote an inner-shell electron to an excited bound state, often denoted as resonant Auger state (RAS), which can decay either through an Auger transition or by the emission of an X-ray photon. This process was first reported by Brown [1] and since then a large number of investigations have taken place (see, for example, [2] and references there in).

Under excitation by the strong radiation fields, now available from free electron laser (FEL) sources [3-7], the situation becomes considerably different. Relevant studies in the context of strong laser fields have been reported quite early [8] and in response to recent developments in the x-ray wavelength regime a number of theoretical and experimental works have already appeared [7,9-13]. In the simplest situation, Rohringer and Santra in Ref. [9] have studied the single-photon excitation of a neon $K$-shell electron to a RAS by an x-ray field and a multipeak Auger electron spectrum (AES) is obtained for the fields they considered.

In this work, we examine the AES and the ionic yields in the case where a normal Auger process takes place (as opposed to the RAS process) which involves photoionization of the neutral from the $K$ shell, followed by an Auger decay of the singly charged hole system to the doubly charged ion. We demonstrate the emergence of ac-Stark splitting (also known as Autler-Townes splitting [14]) of the Auger resonance, resulting from strong Rabi coupling of the apposite states in the resulting $\mathrm{Ne}^{2+}$ ion. In contrast to RAS line-shape modification [9], the unusual phenomenon here is that the ac-Stark splitting is manifested in the kinetic spectrum of the Auger electron, while strong Rabi coupling occurs to one of the $K$-shell electrons of the doubly charged ion. This effect requires an explanation on the basis of a two-electron representation of the Auger-electron ejection, instead of an ambiguous one-electron picture implied

\footnotetext{
*Corresponding author: Lampros.Nikolopoulos@dcu.ie
}

by the Rabi-type oscillation of an inner electron. A detailed study of the two-electron representation, in a different context and formalism but similar physical background, can be found in Ref. [15].

We have chosen as the target of our study neutral neon in its ground state $|G\rangle=\left|\mathrm{Ne}\left(1 s^{2} 2 s^{2} 2 p^{6},{ }^{1} S_{0}\right)\right\rangle$ and consider radiation with a photon energy of approximately $\omega=908 \mathrm{eV}$ (see Fig. 1). The ionization potential for the creation of a $K$-shell hole $|i\rangle=\left|\mathrm{Ne}^{+}\left(1 s 2 s^{2} 2 p^{6},{ }^{1} P\right)\right\rangle$ is $E^{(i)}=870 \mathrm{eV}$. Note that all the energies of the neon states are given relative to the neutral neon ground state. Thus, the $\mathrm{x}$-ray radiation will eject a $K$-shell photoelectron with a kinetic energy of approximately $\varepsilon_{i}=38 \mathrm{eV}$. Ionization from the outer shells is also possible but with much lower probability [16]. The generated $K$-shell hole $\mathrm{Ne}^{+}$will decay by filling the $1 s$ vacancy, predominantly to the doubly charged neon state $|a\rangle=\mid \mathrm{Ne}^{2+}\left(1 s^{2} 2 s^{2} 2 p^{4}\right.$, $\left.\left.{ }^{1} D_{2}\right)\right\rangle$ with energy $E^{(a)}=65.35 \mathrm{eV}$, and Auger decay width $\Gamma_{i a}=0.27 \mathrm{eV}$ [16]. In addition, the $\mathrm{Ne}^{+}$ion with one $K$-shell hole can also decay through fluorescence by emitting $\mathrm{x}$-ray radiation [16]. This channel is about 55 times weaker than the Auger decay rate. Let us designate the sum of all decay channels of the $|i\rangle$ state as $\Gamma_{i}$. Normally this Auger transition of the singly charged ion to the doubly charged ground state is accompanied by the ejection of an electron (Auger electron) with a kinetic energy $\varepsilon_{a}^{(0)}=E^{(i)}-E^{(a)} \sim 804.65 \mathrm{eV}$ and a Lorentzian line profile. In the present case we have chosen the photon energy to match the $\mathrm{Ne}^{2+}\left(1 s^{-1}-3 p\right)$ transition energy. Around this energy there is a manifold of excited states which we denote collectively as $\left|a^{\prime}\right\rangle$. We show in Table I those states which lie within a $1 \mathrm{eV}$ band around the excitation photon energy. While the Auger state $|i\rangle$ decays with a lifetime of about $\Gamma_{i a}^{-1} \sim 2.44 \mathrm{fs}$, the field through a Rabi-type transition creates a coherent superposition of the ionic ground state $|a\rangle$ and all accessible excited states $\left|a^{\prime}\right\rangle$. This Rabi oscillation of the $K$-shell electron between the bound states of the $\mathrm{Ne}^{2+}$ ion will induce an ac-Stark splitting manifested in the kinetic energy of the ejected Auger electron. We should note that no post-collision interaction with the photoelectron is taken into account, as the energy of the photoelectron is too large for such an effect to contribute in the Auger's electron spectrum (and vice versa). To complete the picture, the excited states $\left|a^{\prime}\right\rangle$ decay either through an additional Auger process $\left(\Gamma_{a^{\prime}}\right)$ or through further ionization by absorbing one more photon $\left(\gamma_{a^{\prime}}\right)$. Finally, direct creation of a double core-hole $\mathrm{Ne}^{2+}$ of the neutral through photoabsorption is not considered since the double $K$-shell ionization energy is $1863 \mathrm{eV}$ [17]. 


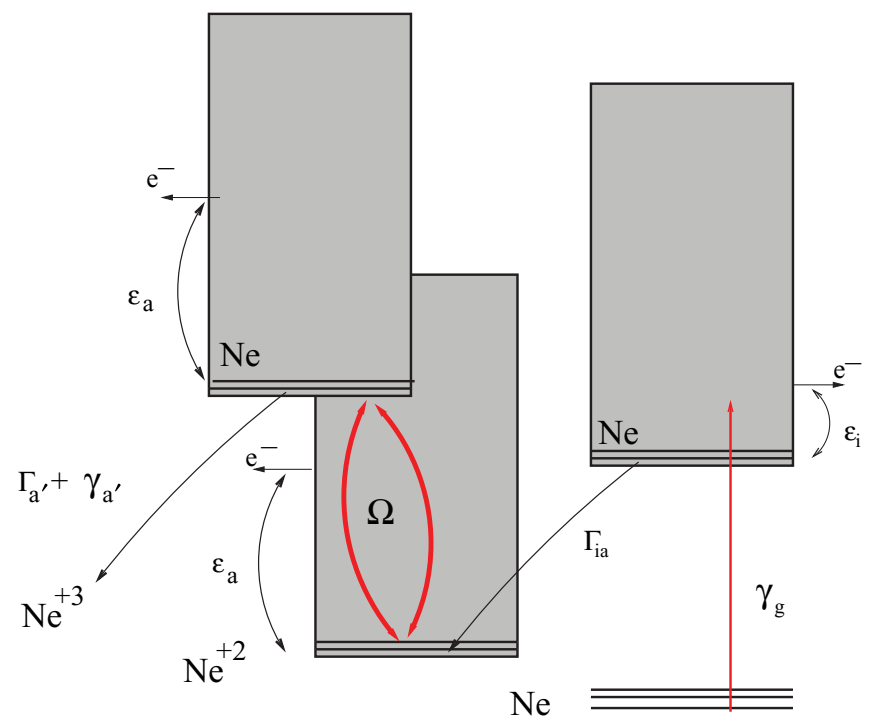

FIG. 1. (Color online) Schematic figure of the dominant channels involved in the interaction of neon with an x-ray field of frequency approximately $908.06 \mathrm{eV}$, chosen to match the $\mathrm{Ne}^{2+}\left(1 s^{-1}-3 p\right)$ transition energy.

We discuss below the manner in which the AES is modified due to the Rabi coupling of the $\mathrm{Ne}^{2+}\left(1 s^{-1}-3 p\right)$ states and study its behavior in a quantitative manner. To facilitate the interpretation of the results, we note at this point that for a modification of the Auger line to appear, many Rabi oscillations should occur within the relevant Auger lifetime. Equivalently, in the energy domain, it is required that the energy separation of the Auger-line splitting (roughly equal to Rabi coupling strength) should be larger than the Auger decay width or the x-ray bandwidth, whichever is larger. Of course the detailed properties of the system and the field will matter as well; however, the rule of thumb, as expressed above, will be in general true.

The structure of the paper is as follows. In Sec. II we present the theoretical formulation and develop the description of the processes in terms of a time-dependent density matrix system of equations. In Sec. III we show some of the results of our present study in the case of a single-mode coherent field. In the final section (Sec. IV) we summarize our findings and discuss, very briefly, two aspects of the problem that are necessary

TABLE I. Transitions from the $|a\rangle=\mathrm{Ne}^{2+}\left({ }^{1} D_{2}\right)$ ground state to its excited states $\mathrm{Ne}^{2+}\left(1 s^{-1}-3 p\right)$ around the photon frequency $\omega=908 \mathrm{eV}$. The fourth column shows the corresponding oscillator strengths. The data are calculated using the Cowan suites of codes [21].

\begin{tabular}{lccl}
\hline \hline$\left|a^{\prime}\right\rangle$ & $E_{a^{\prime}}(\mathrm{eV})$ & $\mathrm{Ne}^{2+}\left(1 s^{1} 2 s^{2}\right)$ & $10^{-2} g f_{a a^{\prime}}$ \\
\hline 1 & 907.75 & $\left(2 p^{4},{ }^{1} D\right)^{2} D\left(3 p^{1}\right)^{1} P_{1}$ & 2.3338 \\
2 & 907.90 & $\left(2 p^{4},{ }^{3} P\right)^{2} P\left(3 p^{1}\right)^{3} P_{1}$ & 0.20991 \\
3 & 908.06 & $\left(2 p^{4},{ }^{1} D\right)^{2} D\left(3 p^{1}\right)^{1} F_{3}$ & 8.1881 \\
4 & 908.48 & $\left(2 p^{4},{ }^{3} P\right)^{2} P\left(3 p^{1}\right)^{3} D_{3}$ & 0.13141 \\
5 & 908.51 & $\left(2 p^{4},{ }^{1} D\right)^{2} D\left(3 p^{1}\right)^{3} D_{2}$ & 0.23322 \\
6 & 908.49 & $\left(2 p^{4},{ }^{3} P\right)^{2} P\left(3 p^{1}\right)^{1} D_{2}$ & 4.4888 \\
7 & 908.78 & $\left(2 p^{4},{ }^{1} D\right)^{2} D\left(3 p^{1}\right)^{1} D_{2}$ & 1.2714 \\
\hline \hline
\end{tabular}

to put the description of the problem closer to the actual experimental conditions; namely, the fluctuations present in a FEL field and the field's spatial dependence.

\section{THEORETICAL FORMULATION OF THE DENSITY MATRIX EQUATIONS}

The density operator of the system is obtained on the basis of $|G\rangle,|I\rangle,|A\rangle,\left|A^{\prime}\right\rangle,|R\rangle,\left|F_{i}\right\rangle, i=1,2$ states. The state $|G\rangle$, with energy $E^{(g)}$, represents the neon ground state. The state $|I\rangle=\left|i ; \varepsilon_{i}\right\rangle$, with energy $E_{i}=E^{(i)}+\varepsilon_{i}$, represents the $K$-shell hole $\mathrm{Ne}^{+}$(state $|i\rangle$ with energy $E^{(i)}$ ) and the photoejected electron $\left|\varepsilon_{i}\right\rangle$ having kinetic energy $\varepsilon_{i}$. The state $|A\rangle=\left|a ; \varepsilon_{a}, \varepsilon_{i a}\right\rangle$, with energy $E_{a}=E^{(a)}+\varepsilon_{a}+\varepsilon_{i a}$, represents the $\mathrm{Ne}^{2+}$ ion in its ground state (state $|a\rangle$ with energy $E^{(a)}$ ) with an ejected Auger electron of kinetic energy $\varepsilon_{a}$ and the photoelectron having now kinetic energy $\varepsilon_{i a}$. Similarly, the state $\left|A^{\prime}\right\rangle=\left|a^{\prime} ; \varepsilon_{a^{\prime}}, \varepsilon_{i a^{\prime}}\right\rangle$, with energy $E_{a^{\prime}}=E^{\left(a^{\prime}\right)}+\varepsilon_{a^{\prime}}+\varepsilon_{i a^{\prime}}$, represents the excited state of $\mathrm{Ne}^{2+}$ (state $\left|a^{\prime}\right\rangle$ with energy $\left.E^{\left(a^{\prime}\right)}\right)$ with the Auger electron having kinetic energy $\varepsilon_{a^{\prime}}$ and the photoelectron having now kinetic energy $\varepsilon_{i a^{\prime}}$. It should be noted that in the definition of the photoelectron and Auger-electron states the appropriate angular momentum quantum numbers, as they result from electric dipole and Auger transition rules, are included. In addition, we also take into account the possibility of the involvement of further decay modes. In the present case, the $K$-shell hole $\mathrm{Ne}^{+}|i\rangle$ can decay through fluorescence to $\mathrm{Ne}^{+}\left(1 s^{2} 2 s^{2} 2 p^{5}\right)$, denoted here as $|R\rangle$. Moreover, the excited states $\left|A^{\prime}\right\rangle$ may further decay either through an Auger transition to $\mathrm{Ne}^{3+}\left(1 s^{2} 2 s^{2} 2 p^{3}\right)$ denoted as $\left|F_{1}\right\rangle$ or through further photoionization to hollow $K$-shell $\mathrm{Ne}^{3+}\left(1 s^{1} 2 s^{2} 2 p^{4}\right)$, denoted as $\left|F_{2}\right\rangle$. The equations of motion for the density matrix elements are obtained from the Liouville equation $i \dot{\rho}(t)=[H(t), \rho(t)]$ with $\hat{H}(t)=\hat{H}^{0}+\hat{V}+\hat{D}(t)$, $\hat{H}^{0}$ being the field-free Hamiltonian of neon, $\hat{V}$ is the electronelectron interaction operator, and $\hat{D}(t)$ is the $\mathrm{x}$-ray field-atomic dipole interaction operator. Inserting the above states into the Liouville equation we obtain

$$
\begin{aligned}
& \dot{\rho}_{G G}(t)=2 \operatorname{Im} \bigvee_{I} D_{G I} \rho_{I G}, \\
\dot{\rho}_{I I}(t)= & 2 \operatorname{Im}\left[D_{I G} \rho_{G I}\right]+2 \operatorname{Im} \bigvee_{A} V_{I A} \rho_{A I} \\
+ & 2 \operatorname{Im} \bigvee_{R} D_{I R} \rho_{R I}, \\
\dot{\rho}_{A A}(t)= & 2 \operatorname{Im}\left[V_{A I} \rho_{I A}\right]+2 \operatorname{Im}\left[D_{A A^{\prime}} \rho_{A^{\prime} A}\right], \\
\dot{\rho}_{A^{\prime} A^{\prime}}(t)= & -2 \operatorname{Im}\left[D_{A A^{\prime}} \rho_{A^{\prime} A}\right]+2 \operatorname{Im} \bigvee_{F_{1}} V_{A^{\prime} F_{1}} \rho_{F_{1} A^{\prime}} \\
& +2 \operatorname{Im} \sum_{F_{2}} D_{A^{\prime} F_{2}} \rho_{F_{2} A^{\prime}}, \\
i \dot{\rho}_{A A^{\prime}}(t)= & E_{A A^{\prime}} \rho_{A A^{\prime}}+D_{A A^{\prime}}\left(\rho_{A^{\prime} A^{\prime}}-\rho_{A A}\right)+V_{A I} \rho_{I A^{\prime}} \\
& -\sum_{F_{1}} \rho_{A F_{1}} V_{F_{1} A^{\prime}}-\sum_{F_{2}} \rho_{A F_{2}} D_{F_{2} A^{\prime}}, \\
& i \dot{\rho}_{G I}(t)=\ldots,
\end{aligned}
$$


In the above expressions $\rho_{K L}, K, L=G, I, A, A^{\prime}, R, F_{1}, F_{2}$ are the density matrix elements of the involved states while $D_{K L}$ and $V_{K L}$ represent electric dipole and Auger (intraatomic) transitions between the states $K, L$, respectively. More specifically, the quantities $D_{G I}, V_{I A}, D_{I R}, D_{A A^{\prime}}, V_{A^{\prime} F_{1}}, D_{A^{\prime} F_{2}}$ represent multielectron electric dipole $(D)$ and Auger $(V)$ transition matrix elements. Within the present context we do not take into account any post-collision effects between the photo and Auger electrons as their contribution are expected to be negligible for the considered kinetic energies as discussed in Ref. [18]. This assumption allows for a simplification of the transition matrix elements as, for example, for $V_{I A}=$ $\left\langle i, \varepsilon_{i}|\hat{V}| a, \varepsilon_{a}, \varepsilon_{i a}\right\rangle$, which reduces to $V_{I A}=\langle i|\hat{V}| a\rangle\left\langle\varepsilon_{i a} \mid \varepsilon_{i}\right\rangle=$ $V_{i a} \delta\left(\varepsilon_{i a}-\varepsilon_{i}\right)$. Along the same lines the dipole transition $D_{A A^{\prime}}=\left\langle a, \varepsilon_{a}, \varepsilon_{i a}|\hat{D}| a^{\prime}, \varepsilon_{a}^{\prime}, \varepsilon_{i a^{\prime}}\right\rangle$ is approximated as $D_{A A^{\prime}}=$ $\left\langle a|\hat{D}| a^{\prime}\right\rangle\left\langle\varepsilon_{a} \mid \varepsilon_{a^{\prime}}\right\rangle\left\langle\varepsilon_{i a} \mid \varepsilon_{i a^{\prime}}\right\rangle=d_{a a^{\prime}} \delta\left(\varepsilon_{a}-\varepsilon_{a^{\prime}}\right) \delta\left(\varepsilon_{i a}-\varepsilon_{i a^{\prime}}\right)$. A detailed discussion of the dimensional reduction of these special kind of continuum-continuum matrix elements can be found in the Appendix of Ref. [15]. The summations involved here imply integration over the appropriate continua. As the total number of independent equations is 28 , we do not present the explicit expressions for the evolution of the remaining density matrix elements as they are not essential at this stage.

The density matrix equations are a system of coupled integrodifferential equations which are not amenable to an easy solution even by numerical means, as it includes integration over multidimensional continua. It is thus our purpose here to transform the above system of equations into a more tractable form. To this end, we adiabatically eliminate the density matrix elements which are involved in the integrations over the respective continua of the states. The procedure for adiabatically eliminating these continua is a standard technique applied to describe the influence of a system with infinite degrees of freedom onto a system with a small number of degrees and appears in many different contexts (see, e.g., [19]). Here the reduced system is the one described by $|G\rangle,|I\rangle,|A\rangle$ and $\left|A^{\prime}\right\rangle$ while $|R\rangle,\left|F_{i}\right\rangle, i=1,2$ represent the dissipative environment. Within the present context of atomic continua, some of the details can also be found in Ref. [20]. To proceed further, the radiation field is expressed as $\mathbf{E}(t)=\hat{e}\left[\mathcal{E}(t) e^{i \omega t}+\mathcal{E}^{\star}(t) e^{-i \omega t}\right] / 2$ with $\hat{e}$ its polarization vector and we transform to slowly varying variables by defining $\sigma_{k l}=\rho_{K L} e^{-i n \omega t}, n=0, \pm 1, \pm 2, \pm 3$, where $n$ is chosen so that $n \omega$ has the closest possible value to $E_{K}-E_{L}$. With the latter transformation we remove from the coherences the fast oscillation part of their evolution due to the frequency of the field (this is justified since for a frequency of $1 \mathrm{keV}$ the field period is of the order of 4 as and all other time scales set by photoionization and Auger widths are of the order of $1 \mathrm{fs}-1000 \mathrm{as}$ ). Given that the radiation is in the form of a pulse, we have kept the slowly varying envelope $\mathcal{E}(t)$ which in addition may describe the stochastic properties of the field under consideration. This is however a problem which requires special care and postpone its discussion for now. In the present case we assume a fully coherent singlemode, Fourier transform-limited, field. Then, by employing the rotating wave approximation (RWA) and keeping only the terms proportional to the first order of the electric field, after tedious but straightforward manipulation we end up to the following set of equations for the reduced density matrix elements:

$$
\begin{aligned}
& \dot{\sigma}_{g g}(t)=-\gamma_{g} \sigma_{g g}, \\
& \dot{\sigma}_{i i}\left(\varepsilon_{i}, t\right)=-\Gamma_{i} \sigma_{i i}+\operatorname{Im}\left[\Omega_{i g}^{\star} \sigma_{g i}\right], \\
& \dot{\sigma}_{a a}\left(\varepsilon_{i}, \varepsilon_{a}, t\right)=-\operatorname{Im}\left[\Omega_{a^{\prime} a}^{\star} \sigma_{a a^{\prime}}\right]+2 \operatorname{Im}\left[V_{a i} \sigma_{i a}\right], \\
& \dot{\sigma}_{a^{\prime} a^{\prime}}\left(\varepsilon_{i}, \varepsilon_{a}, t\right)=-\bar{\gamma}_{a^{\prime}} \sigma_{a^{\prime} a^{\prime}}+\operatorname{Im}\left[\Omega_{a^{\prime} a}^{\star} \sigma_{a a^{\prime}}\right], \\
& i \dot{\sigma}_{a a^{\prime}}\left(\varepsilon_{i}, \varepsilon_{a}, t\right)=\left(E_{a a^{\prime}}+\omega-i \frac{\bar{\gamma}_{a^{\prime}}}{2}\right) \sigma_{a a^{\prime}} \\
& +\frac{\Omega_{a a^{\prime}}}{2}\left(\sigma_{a^{\prime} a^{\prime}}-\sigma_{a a}\right)+V_{a i} \sigma_{i a^{\prime}}, \\
& i \dot{\sigma}_{g i}\left(\varepsilon_{i}, t\right)=\left(E_{g i}+\omega-i \frac{\gamma_{g}+\Gamma_{i}}{2}\right) \sigma_{g i}-\frac{1}{2} \Omega_{g i} \sigma_{g g}, \\
& i \dot{\sigma}_{i a}\left(\varepsilon_{i}, \varepsilon_{a}, t\right)=\left(E_{i a}-i \frac{\Gamma_{i}}{2}\right) \sigma_{i a}+\frac{1}{2} \Omega_{i g}^{*} \sigma_{g a} \\
& -\frac{1}{2} \Omega_{a^{\prime} a}^{*} \sigma_{i a^{\prime}}-V_{i a} \sigma_{i i}, \\
& i \dot{\sigma}_{i a^{\prime}}\left(\varepsilon_{i}, \varepsilon_{a}, t\right)=\left(E_{i a^{\prime}}+\omega-i \frac{\Gamma_{i}+\bar{\gamma}_{a^{\prime}}}{2}\right) \sigma_{i a^{\prime}} \\
& +\frac{1}{2} \Omega_{i g}^{*} \sigma_{g a^{\prime}}-\frac{1}{2} \Omega_{a a^{\prime}} \sigma_{i a}, \\
& i \dot{\sigma}_{g a}\left(\varepsilon_{i}, \varepsilon_{a}, t\right)=\left(E_{g a}+\omega-i \frac{\gamma_{g}}{2}\right) \sigma_{g a}-\frac{1}{2} \Omega_{a^{\prime} a}^{*} \sigma_{g a^{\prime}}-V_{i a} \sigma_{g i} \text {, } \\
& i \dot{\sigma}_{g a^{\prime}}\left(\varepsilon_{i}, \varepsilon_{a}, t\right)=\left(E_{g a^{\prime}}+2 \omega-i \frac{\gamma_{g}+\bar{\gamma}_{a^{\prime}}}{2}\right) \sigma_{g a^{\prime}}-\frac{1}{2} \Omega_{a a^{\prime}} \sigma_{g a},
\end{aligned}
$$

where $\Omega_{g i}\left(\varepsilon_{i}, t\right)=\left\langle g|\hat{D}| i, \varepsilon_{i}\right\rangle \mathcal{E}(t) \quad$ and $\quad \Omega_{a a^{\prime}}(t)=d_{a a^{\prime}} \mathcal{E}(t)$. With $\gamma_{g}(t)=2 \pi \int d \varepsilon_{i}\left|\Omega_{g i}\left(\varepsilon_{i}, t\right)\right|^{2}$ we denote the photoionization width of the neon ground states relative to the $\mathrm{Ne}^{+} K$-shell hole state $|i\rangle$, while $\bar{\gamma}_{a^{\prime}}=\Gamma_{a^{\prime}}+\gamma_{a^{\prime}}(t)$ is the sum of the Auger decay width to states $\left|F_{1}\right\rangle\left(\Gamma_{a^{\prime}}\right)$ and the photoionization width to states $\left|F_{2}\right\rangle\left(\gamma_{a^{\prime}}\right)$ of the excited states $\left|a^{\prime}\right\rangle$. In addition, $\Gamma_{i}=$ $\Gamma_{i a}+\gamma_{r}$ is the sum of the decay width of the ionic state $|i\rangle$ through Auger decay to states $|a\rangle$ and through fluorescence to states $|R\rangle$. The quantity $V_{i a}$ represents the strength of the Auger transition of the hole state $|i\rangle$ to the particular ionic ground state $|a\rangle$. Therefore, the quantity $\Gamma_{i a}$ is expressed as $\Gamma_{i a}=$ $2 \pi\left|V_{i a}\right|^{2}$. Finally, the energy differences in the above equations now include all the shifts associated with the Auger and dipole couplings of the relevant states with continuum states $E_{k l} \equiv E_{k}+S_{k}-\left(E_{l}+S_{l}\right), k, l=g, i, a^{\prime}$ with $S_{i}=S_{i a}+S_{i r}$ and $S_{a^{\prime}}=S_{a^{\prime} f_{1}}+S_{a^{\prime} f_{2}}$. The exact definition of the shifts and widths are as below:

$$
\begin{aligned}
S_{g}-i \frac{\gamma_{g}}{2} & =\lim _{\eta \rightarrow 0} \int d E_{I} \frac{\left|D_{G I}\right|^{2}}{E_{G}+\omega-E_{I}+i \eta}, \\
S_{i a}-i \frac{\Gamma_{i a}}{2} & =\lim _{\eta \rightarrow 0} \int d E_{A} \frac{\left|V_{I A}\right|^{2}}{E_{I}-E_{A}+i \eta}, \\
S_{i r}-i \frac{\Gamma_{i r}}{2} & =\lim _{\eta \rightarrow 0} \int d E_{R} \frac{\left|D_{I R}\right|^{2}}{E_{I}-E_{R}+i \eta}, \\
S_{a^{\prime} f_{1}}-i \frac{\Gamma_{a^{\prime}}}{2} & =\lim _{\eta \rightarrow 0} \int d E_{F_{1}} \frac{\left|V_{A^{\prime} F_{1}}\right|^{2}}{E_{A^{\prime}}-E_{F_{1}}+i \eta}, \\
S_{a^{\prime} f_{2}}-i \frac{\gamma_{a^{\prime}}}{2} & =\lim _{\eta \rightarrow 0} \int d E_{F_{2}} \frac{\left|D_{A^{\prime} F_{2}}\right|^{2}}{E_{A^{\prime}}+\omega-E_{F_{2}}+i \eta},
\end{aligned}
$$


where use of the well-known formula $\lim _{\eta \rightarrow 0} 1 /(x+i \eta)=$ $\mathrm{P}(1 / x)-i \pi \delta(x)$ must be made to split up the integrals into their real and imaginary parts.

At this stage, a working set of equations is established and their numerical solution is feasible, provided that all the dynamical parameters of the problem have been calculated beforehand. The approximations leading to this set of equations require careful examination of the appropriate range of radiation intensities. One approximation is to assume that the relevant continua are smooth around the energies of the dressed bound states within an energy range comparable with the Rabicoupling matrix element. This requires that transitions close to ionization thresholds should not be considered. In addition, in the derivation procedure we have ignored terms proportional to the second order of the field, such that $\Omega_{a a^{\prime}}<1$. The latter approximation, given the matrix element $d_{a a^{\prime}} \sim 0.06$ a.u., will restrict the range of the intensities where the working equations are applicable below to $4 \times 10^{18} \mathrm{~W} / \mathrm{cm}^{2}$.

The system of equations that have been derived must, simultaneously, be numerically integrated for all different photoelectron and Auger-electron kinetic energies, so as to provide the populations for $\sigma_{i i}\left(\varepsilon_{i}\right), \sigma_{a a}\left(\varepsilon_{a}, \varepsilon_{i}\right), \sigma_{a^{\prime} a^{\prime}}\left(\varepsilon_{a}, \varepsilon_{i}\right)$ at infinite times. Since in our case we are only interested in the Auger-kinetic energy spectrum and ionization yields regardless of the state of the photoelectron, we must integrate the final populations over the photoelectron kinetic energies and determine the following probabilities: $\sigma_{i i}=\int d \varepsilon_{i} \sigma_{i i}\left(\varepsilon_{i}\right), \sigma_{a a}\left(\varepsilon_{a}\right)=$ $\int d \varepsilon_{i} \sigma_{a a}\left(\varepsilon_{a}, \varepsilon_{i}\right), \sigma_{a^{\prime} a^{\prime}}\left(\varepsilon_{a}\right)=\int d \varepsilon_{i} \sigma_{a^{\prime} a^{\prime}}\left(\varepsilon_{a}, \varepsilon_{i}\right)$. An alternative and more economical way of obtaining the same results is to derive a coarse-grained version of the present equations for these reduced, averaged over the photoelectron energies, density matrix elements. In addition, the reduced set of the density matrix equations is amenable to further manipulation as it allows for the derivation of analytical expressions for long pulses or their averaging for stochastic pulses. Thus, the new reduced set of equations is obtained by first setting all the derivatives of the coherences equal to zero [except $\left.\dot{\sigma}_{a a^{\prime}}\left(\varepsilon_{a}, \varepsilon_{i}, t\right)\right]$ and then integrating over the photoelectron energy $\varepsilon_{i}$. To demonstrate the reasoning of setting the derivatives of the coherences to zero, we work out the evolution equation of $\sigma_{g i}\left(\epsilon_{i}, t\right)$ coherence [Eq. (1f)]. We integrate Eq. (1f) in an interval $t, t+\tau$ with $\tau \ll \Omega_{a a^{\prime}}^{-1}, \gamma_{g}^{-1}, \Gamma_{i}^{-1}$ and we obtain

$$
\begin{aligned}
& i\left[\sigma_{g i}\left(\varepsilon_{i}, t+\tau\right)-\sigma_{g i}\left(\varepsilon_{i}, t\right)\right] \\
& =\left[E_{g i}(\tau)+\omega-i \frac{\gamma_{g}(\tau)+\Gamma_{i}}{2}\right] \int_{t}^{t+\tau} d t^{\prime} \sigma_{g i}\left(\varepsilon_{i}, t^{\prime}\right) \\
& \quad-\frac{\Omega_{g i}\left(\varepsilon_{i}, \tau\right)}{2} \int_{t}^{t+\tau} d t^{\prime} \sigma_{g g}\left(t^{\prime}\right) .
\end{aligned}
$$

The ionization width $\gamma_{g}(\tau)$, the dipole $\Omega_{g i}\left(\varepsilon_{i}, \tau\right)$, and the $S_{g}(\tau), S_{i}(\tau)$ ac-Stark shifts that are included in the definition of $E_{g i}(\tau)$ were removed from the integral as their value does not change much between $t$ and $t+\tau$ as a result of the slowly varying transformation of the variables. Since it will always be $\left|\sigma_{g i}\left(\varepsilon_{i}, t\right)\right| \ll \sigma_{g g}(t)$, we can neglect the left-hand side and obtain the "coarse-grained" time average of $\sigma_{g i}\left(\varepsilon_{i}, t\right)$ as

$$
\bar{\sigma}_{g i}\left(\varepsilon_{i}, \tau\right)=\frac{\Omega_{g i}\left(\varepsilon_{i}, \tau\right) / 2}{E_{g i}(\tau)+\omega-i\left[\gamma_{g}(\tau)+\Gamma_{i}\right] / 2} \bar{\sigma}_{g g}(\tau),
$$

where $\quad \bar{\sigma}_{g i}\left(\varepsilon_{i}, \tau\right) \equiv \int_{t}^{t+\tau} d t^{\prime} \sigma_{g i}\left(\varepsilon_{i}, t^{\prime}\right) / \tau \quad$ and $\quad \bar{\sigma}_{g g}(\tau) \equiv$ $\int_{t}^{t+\tau} d t^{\prime} \sigma_{g g}\left(t^{\prime}\right) / \tau$. Thus, by setting the derivative of the coherence to zero, effectively leads to a coarse-grained value for the coherence which follows adiabatically the ground state population. At this stage, integrating Eq. (1b) over time (in an interval $[t, t+\tau])$ and the photoelectron energy $\varepsilon_{i}$ we obtain

$$
\begin{aligned}
\dot{\sigma}_{i i}(\tau)= & -\Gamma_{i} \sigma_{i i}(\tau) \\
& +\operatorname{Im} \int d \varepsilon_{i} \frac{\left|\Omega_{g i}\left(\varepsilon_{i}, \tau\right)\right|^{2} / 2}{E_{g i}(\tau)+\omega-i\left[\gamma_{g}(\tau)+\Gamma_{i}\right] / 2} \sigma_{g g}(\tau) \\
= & -\Gamma_{i} \sigma_{i i}(\tau)+\gamma_{g}(\tau) \sigma_{g g}(\tau),
\end{aligned}
$$

where $\sigma_{i i}(\tau)=\int d \varepsilon_{i} \bar{\sigma}_{i i}\left(\varepsilon_{i}, \tau\right)$ and $\bar{\sigma}_{i i\left(\varepsilon_{i}, \tau\right)}=\int_{t}^{t+\tau} d t^{\prime} \sigma_{i i}\left(\varepsilon_{i}, t^{\prime}\right)$. To evaluate the integral we have assumed that $\Omega_{g i}\left(\varepsilon_{i}, \tau\right)$ is smooth over an energy range equal to the radiation's bandwidth (far from resonance structures in the continuum or ionization thresholds). Then by expressing $E_{g i}$ as $E_{g i}(\tau)=E^{(g)}+S_{g}(\tau)-E^{(i)}-S_{i}(\tau)-\varepsilon_{i}$ we have $\operatorname{Im} \int d \varepsilon_{i}\left|\Omega_{g i}\left(\varepsilon_{i}, \tau\right)\right|^{2} /\left\{E_{g i}(\tau)+\omega-i\left[\gamma_{g}(\tau)+\Gamma_{i}\right] / 2\right\} \sim$ $\pi\left|\Omega_{g i}\left(\varepsilon_{i}=E^{(i)}+S_{i}-E^{(g)}-S_{g}, \tau\right)\right|^{2}=2 \gamma_{g}(\tau)$.

As the derivation is quite long and detailed for the remaining coherences, we give here only the final result for the reduced (coarse-grained) set of density matrix equations. These reduced equations are obtained working along similar lines as for the derivation of Eq. (3). In this derivation we ignore the photoionization of the excited $\left|a^{\prime}\right\rangle\left(\gamma_{a^{\prime}}=0\right)$, the photon energies and the intensities considered is expected to be much less than the Auger decay transition represented by $\Gamma_{a^{\prime}}$. Therefore, after setting all the derivatives of the coherences equal to zero [Eqs. (1f), (1g), (1h), (1i), and (1j)], we solve for the coherences and substitute their values into Eqs. (1c), (1d), and (1e). Then we integrate our equations over the photoelectron's kinetic energy and obtain the coarse-grained (also changing $\tau \rightarrow t$ ) set of density matrix equations,

$$
\begin{gathered}
\dot{\sigma}_{g g}(t)=-\gamma_{g} \sigma_{g g} \\
\dot{\sigma}_{i i}(t)=-\Gamma_{i} \sigma_{i i}+\gamma_{g} \sigma_{g g} \\
\dot{\sigma}_{a a}\left(\varepsilon_{a}, t\right)=-\operatorname{Im}\left[\Omega_{a^{\prime}}^{\star} \sigma_{a a^{\prime}}\right] \\
+\operatorname{Im}\left[\left(\Delta_{a}+\delta_{a^{\prime}}-i \frac{\Gamma_{i}+\Gamma_{a^{\prime}}}{2}\right)\left(\Omega_{a^{\prime}}^{+}-\Omega_{a^{\prime}}^{-}\right)\right] \sigma_{i i} \\
\dot{\sigma}_{a^{\prime} a^{\prime}}\left(\varepsilon_{a}, t\right)=-\Gamma_{a^{\prime}} \sigma_{a^{\prime} a^{\prime}}+\operatorname{Im}\left[\Omega_{a^{\prime}}^{\star} \sigma_{a a^{\prime}}\right] \\
i \dot{\sigma}_{a a^{\prime}}\left(\varepsilon_{a}, t\right)=\left(\delta_{a^{\prime}}-i \frac{\Gamma_{a^{\prime}}}{2}\right) \sigma_{a a^{\prime}}-\frac{\Omega_{a^{\prime}}}{2}\left(\sigma_{a^{\prime} a^{\prime}}-\sigma_{a a}\right) \\
+\frac{\Omega_{a^{\prime}}}{4}\left(\Omega_{a^{\prime}}^{+}-\Omega_{a^{\prime}}^{-}\right) \sigma_{i i}
\end{gathered}
$$

The dynamics of the process are governed by the ionization width of the neutral target $\gamma_{g}(t)$, the core Rabicoupling $\Omega_{a^{\prime}}(t)$, the intra-atomic decay rates $\Gamma_{i}, \Gamma_{a^{\prime}}$, the Auger-field induced couplings $\Omega_{a^{\prime}}^{ \pm}(t)$, and the Auger $\Delta_{a}(t)=$ $E_{i}-\left(E_{a}+\varepsilon_{a}\right)$ and field $\delta_{a^{\prime}}(t)=\left(E_{a}+\omega\right)-E_{a^{\prime}}$ detunings. Note that for notational simplicity we denote the core Rabi coupling as $\Omega_{a^{\prime}}=\Omega_{a a^{\prime}}=\Omega_{a^{\prime} a}$. We have defined $\Omega_{a^{\prime}}^{ \pm}(t)=$ $2\left|V_{i a}\right|^{2} /\left(\Delta_{a^{\prime}}^{ \pm} \bar{\Omega}_{a^{\prime}}\right)$ with $\Delta_{a^{\prime}}^{ \pm}(t)=\epsilon_{a}-\epsilon_{a}^{ \pm}+i \gamma_{a}^{ \pm}(t) / 2, \epsilon_{a}^{ \pm}(t)=$ $\epsilon_{a}^{(0)}+\left[\delta_{a^{\prime}} \mp \bar{\Omega}_{a^{\prime}}^{(r)}(t)\right] / 2$, and $\gamma_{a}^{ \pm}(t)=\Gamma_{i}+\left[\Gamma_{a^{\prime}} \pm \bar{\Omega}_{a^{\prime}}^{(i)}(t)\right] / 2$. 
The real quantities $\bar{\Omega}_{a^{\prime}}^{(r)}$ and $\bar{\Omega}_{a^{\prime}}^{(i)}$ are defined in terms of the generalized Rabi frequency:

$$
\bar{\Omega}_{a^{\prime}}(t)=\bar{\Omega}_{a^{\prime}}^{(r)}+i \frac{\bar{\Omega}_{a^{\prime}}^{(i)}}{2}=\sqrt{\left(\delta_{a^{\prime}}-i \frac{\Gamma_{a^{\prime}}}{2}\right)^{2}+4\left|\Omega_{a^{\prime}}\right|^{2}} .
$$

The AES, at detection time, is obtained by adding the contributions from the ground $|a\rangle$ and excited $\left|a^{\prime}\right\rangle$ states of the doubly ionized neon: $S\left(\varepsilon_{a}\right)=\sum_{j=a, a^{\prime}} \int_{-\infty}^{+\infty} d t^{\prime} \dot{\sigma}_{j j}\left(\varepsilon_{a}, t^{\prime}\right)$. The populations of the states $|a\rangle$ and $\left|a^{\prime}\right\rangle$ are obtained as $p_{j j}(t)=\int_{-\infty}^{+\infty} d t \int d \varepsilon_{i} \dot{\sigma}_{j j}\left(\varepsilon_{a}, t^{\prime}\right), j=a, a^{\prime}$. Although we numerically integrate the above system of density matrix equations (4), the Auger spectra can also be cast in analytical form to a very good approximation. For example, for a nondecaying excited states $\left(\Gamma_{a^{\prime}}=0\right)$ the generalized Rabi frequency becomes a purely real quantity $\bar{\Omega}_{a^{\prime}}=\sqrt{\delta_{a^{\prime}}^{2}+4\left|\Omega_{a^{\prime}}\right|^{2}}$, while the AES become independent on the coherence evolution $\sigma_{a a^{\prime}}$. The analytical approximation consists of considering a pulse of constant amplitude $\mathcal{E}(t)$ which turns the coarsegrained system to a system of ordinary differential equations with constant coefficients. Then the Rabi frequency, ac-Stark level shifts, and the ionization widths become independent in time and in the expression for the AES only the population of $|i\rangle$ is time dependent:

$$
\begin{aligned}
S\left(\varepsilon_{a}\right) & =\int_{-\infty}^{+\infty} d t\left[\dot{\sigma}_{a a}\left(\varepsilon_{a}, t\right)+\dot{\sigma}_{a^{\prime} a^{\prime}}\left(\varepsilon_{a}, t\right)\right] \\
& =\operatorname{Im}\left[\left(\Delta_{a}+\delta_{a^{\prime}}-i \frac{\Gamma_{i}}{2}\right)\left(\Omega_{a^{\prime}}^{+}-\Omega_{a^{\prime}}^{-}\right)\right] \int_{-\infty}^{+\infty} d t \sigma_{i i}(t) .
\end{aligned}
$$

Solving Eqs. (4a) and (4b) for $\sigma_{i i}(t)$ we find that $\int_{-\infty}^{+\infty} d t \sigma_{i i}(t)=1 / \Gamma_{i}$ and after some algebra we end up to the following analytical expression for the AES:

$$
\begin{aligned}
S\left(\varepsilon_{a}\right)= & \frac{\Gamma_{i a}}{4 \pi}\left[\frac{1-\delta_{a^{\prime}} / \bar{\Omega}_{a^{\prime}}}{\left(\varepsilon_{a}-\varepsilon_{a}^{(0)}-\frac{\delta_{a^{\prime}}-\bar{\Omega}_{a^{\prime}}}{2}\right)^{2}+\frac{\Gamma_{i}^{2}}{4}}\right. \\
& \left.+\frac{1+\delta_{a^{\prime}} / \bar{\Omega}_{a^{\prime}}}{\left(\varepsilon_{a}-\varepsilon_{a}^{(0)}-\frac{\delta_{a^{\prime}}+\bar{\Omega}_{a^{\prime}}}{2}\right)^{2}+\frac{\Gamma_{i}^{2}}{4}}\right] .
\end{aligned}
$$

We should note here that the predictions of the analytical expression differ from the numerical solution in that it does not include the transient effects of the physical process which are expected to occur at times of the order of $1 / \Gamma_{i}$.

\section{RESULTS}

In Fig. 2 we show the effect of the field strength in the Auger spectra for $\omega=908.06 \mathrm{eV}$ and assume a pulse envelope $\mathcal{E}(t)=\sin ^{2}(\pi t / T)$, with $T$ being the total pulse duration equal to about 20 times the Auger lifetime $\sim 48.8 \mathrm{fs}$ $(\mathrm{FWHM}=24.4 \mathrm{fs})$. More specifically, in all the following calculations the field included 10754 cycles. The ionization width of the neutral is given by $\gamma_{g}(t)=4.375 \times 10^{-4} \mathcal{E}^{2}(t)$ a.u. In the calculation we only include the $\left|a^{\prime}\right\rangle=\mid \mathrm{Ne}^{2+}\left(1 s^{-1}-3 p\right)$, $\left.{ }^{1} F\right\rangle$ excited state (state 3 of Table I) and assume its decay width to be zero $\left(\Gamma_{a^{\prime}}=0\right)$. For this on-resonance process $\left(\delta_{a^{\prime}}=0\right)$, the Rabi interaction energy is found to be $\Omega_{a^{\prime}}=$ $0.061 \mathcal{E}(t)$ a.u. According to Eq. (6), for peak intensities of $I_{0}=1.0 \times 10^{15}, 1.0 \times 10^{16}, 3.51 \times 10^{16}, 1.0 \times 10^{17}$, and

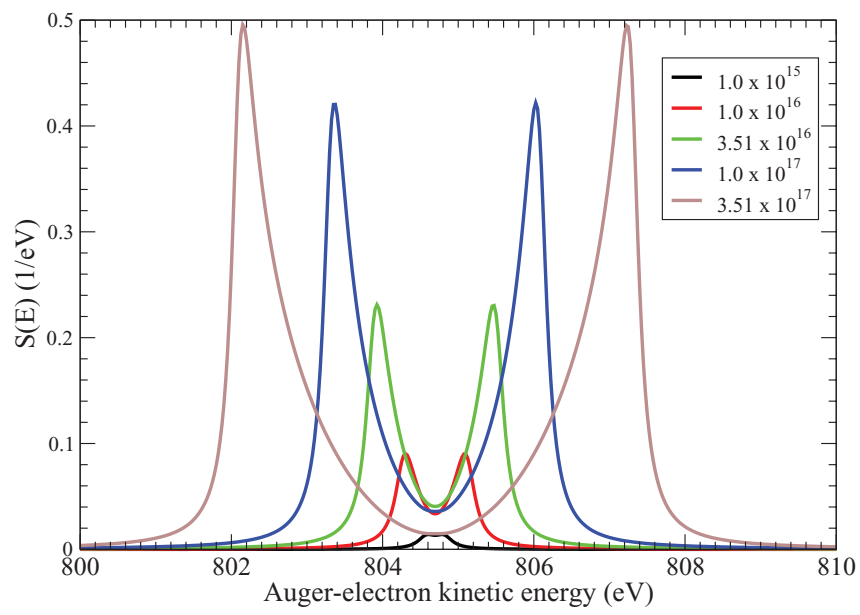

FIG. 2. (Color online) Variation in the AES as the peak intensity of the pulse increases. The field has total duration about $48.8 \mathrm{fs}$ ( $\mathrm{FWHM}=24.4 \mathrm{fs}$ ) and the photon frequency chosen equal to $908.06 \mathrm{eV}$, while $\Gamma_{a^{\prime}}=0$. The inset label give the peak intensity values in units of $\mathrm{W} / \mathrm{cm}^{2}$.

$3.51 \times 10^{17} \mathrm{~W} / \mathrm{cm}^{2}$ peak separations (of equal height) of about $0.28,0.88,1.66,2.79$, and $5.25 \mathrm{eV}$, respectively, should be expected for the AES. Particularly for the lowest intensity of $I_{0}=1.0 \times 10^{15}$ the peak separation is comparable to the Auger decay width $(0.27 \mathrm{eV})$, thus the separation is hardly seen. The latter values coincide with the full numerical solutions, shown in Fig 2. In general, according to the above formula, for on-resonance conditions and long pulses (compared to $1 / \Gamma_{i}$ ) a change of the splitting by a factor around 3.2 should be expected for a change of the peak intensity by one order of magnitude. For off-resonance conditions we have two unequal peaks with energy separation again determined from the generalized Rabi-frequency $\bar{\Omega}_{a^{\prime}}$ and relative height determined from the field detuning $\Delta_{a^{\prime}}$.

Next we turn to the case where the decay channels of the excited core states $\left|a^{\prime}\right\rangle$ are present. We have found that for the intensities and photon energy considered, further photoionization of $\left|a^{\prime}\right\rangle$ is a much weaker channel compared with a RAS transition to $\mathrm{Ne}^{3+}[21,22]$. We take the RAS width to be a large fraction of $\mathrm{Ne}^{3+}\left(1 s 2 p^{2} 2 p^{4}\right) \rightarrow \mathrm{Ne}^{3+}\left(1 s^{2} 2 p^{2} 2 p^{3}\right)$ decay width and assume $\Gamma_{a^{\prime}}=0.156 \mathrm{eV}$ [22]. In this case the lifetime of these excited ionic states $\left|a^{\prime}\right\rangle$ is about $4.12 \mathrm{fs}$, comparable to the Auger decay lifetime of interest here $(\sim 2.45 \mathrm{fs})$ but still much shorter than the pulse duration. Given that the Rabi coupling saturates the $\mathrm{Ne}^{2+}\left(1 s^{-1}-3 p\right)$ transition very quickly (in the sense that their populations are almost equalized) it can be expected that a large portion of the population will very quickly decay to the $\mathrm{Ne}^{3+}$ ion. This behavior is shown in Fig. 3 where the final populations in $\mathrm{Ne}^{2+}$ and $\mathrm{Ne}^{3+}$ are plotted as a function of the peak intensity of the applied x-ray field. Thus for on-resonance cases (solid lines) we see that the majority of the population goes into $\mathrm{Ne}^{3+}$ ion for all intensities considered. On the other hand, if we choose to detune the FEL to a photon energy of $904.06 \mathrm{eV}$ (dashed lines), efficient population of the excited ionic state $\left|a^{\prime}\right\rangle$ is prohibited. This also causes an effective increase in the magnitude of the generalized Rabi frequency according to Eq. (5). In that case, the situation changes dramatically. The relative population 


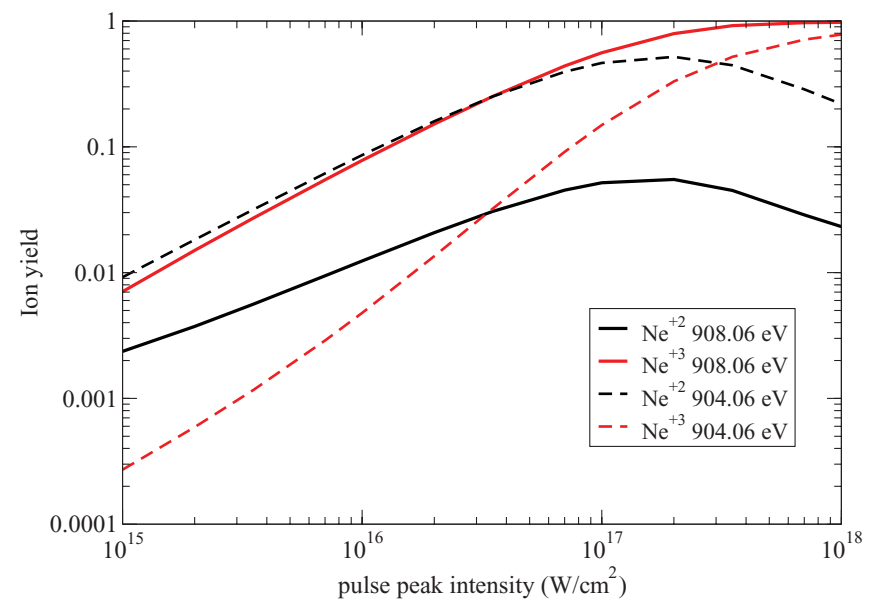

FIG. 3. (Color online) Ionization yields vs the peak intensity of the X-ray field. Field parameters as in Fig. 2 and $\Gamma_{a^{\prime}}=0.156 \mathrm{eV}$. Solid curves refer to $\omega=908.06 \mathrm{eV}$, while the dashed ones to $\omega=$ $904.06 \mathrm{eV}$.

ratio is reversed for low intensity fields $\left(<10^{17} \mathrm{~W} / \mathrm{cm}^{2}\right)$, with the $\mathrm{Ne}^{2+}$ dominating up to higher intensities where the ratio starts to decline in favor of the $\mathrm{Ne}^{3+}$ yield. Thus, it appears that a careful combination of the intensity and the photon energy of the field can control the relative populations of the triply and doubly ionic species of neon.

It is worth noting here that for the present long pulses and for relatively strong fields $\left(>3.5 \times 10^{16} \mathrm{~W} / \mathrm{cm}^{2}\right)$, the depletion of the neutral neon might be significant. For example, for $I_{0}=$ $3.5 \times 10^{16} \mathrm{~W} / \mathrm{cm}^{2}$ the remaining neutral neon is 0.718 , while for ten times stronger field $I_{0}=3.5 \times 10^{17} \mathrm{~W} / \mathrm{cm}^{2}$ decreases to 0.036 . Furthermore, it should also be noted that ionic species higher than triply and doubly ionized neon are not expected to contribute significantly as two- or multiphoton absorption is an unlikely ionization channel, given their ionization potentials and the large photon energy. Finally, a small fraction of $\mathrm{Ne}^{+}$is produced through the fluorescence of the metastable $K$-shell hole $\mathrm{Ne}^{+}$ion.

In Fig. 4 we show the populations of the $\mathrm{Ne}^{2+}$ and $\mathrm{Ne}^{2+}\left(1 s^{-1}-3 p\right)$ excited states as a function of time for a field of peak intensity $1.0 \times 10^{17} \mathrm{~W} / \mathrm{cm}^{2}$. We have considered two different frequencies as in the case of Fig. 3, which represent the on-resonance $(908.06 \mathrm{eV}$, solid lines) and off-resonance (904.06, dashed lines) conditions. From this figure we can see that the populations of $\mathrm{Ne}^{2+}$ and $\mathrm{Ne}^{2+}\left(1 s^{-1}-3 p\right)$ are quickly (almost) equalized, thus allowing for the efficient production of the $\mathrm{Ne}^{3+}$ ion through the Auger decay of the excited $\mathrm{Ne}^{2+}\left(1 s^{-1}-3 p\right)$ state with the ejection of one more electron $\left(\Gamma_{a^{\prime}}=0.156 \mathrm{eV}\right)$. With off-resonance conditions we see that the same populations evolve differently with the amount of $\mathrm{Ne}^{3+}$ that is produced being significantly smaller than the $\mathrm{Ne}^{2+}$ yield for a broad range of intensities. Of course, even in the detuned case, when the intensity becomes higher, the Rabi amplitude will increase accordingly and again the quick transfer from the ground $\mathrm{Ne}^{2+}$ to the excited $\mathrm{Ne}^{2+}\left(1 s^{-1}-3 p\right)$ state will allow the efficient production of the $\mathrm{Ne}^{3+}$ ion. In that case $\mathrm{Ne}^{3+}$ yield will surpass $\mathrm{Ne}^{2+}$ yield. By inspection of Fig. 3, the intensities that this overtaking of $\mathrm{Ne}^{3+}$ takes place are beyond $3.5 \times 10^{17} \mathrm{~W} / \mathrm{cm}^{2}$.

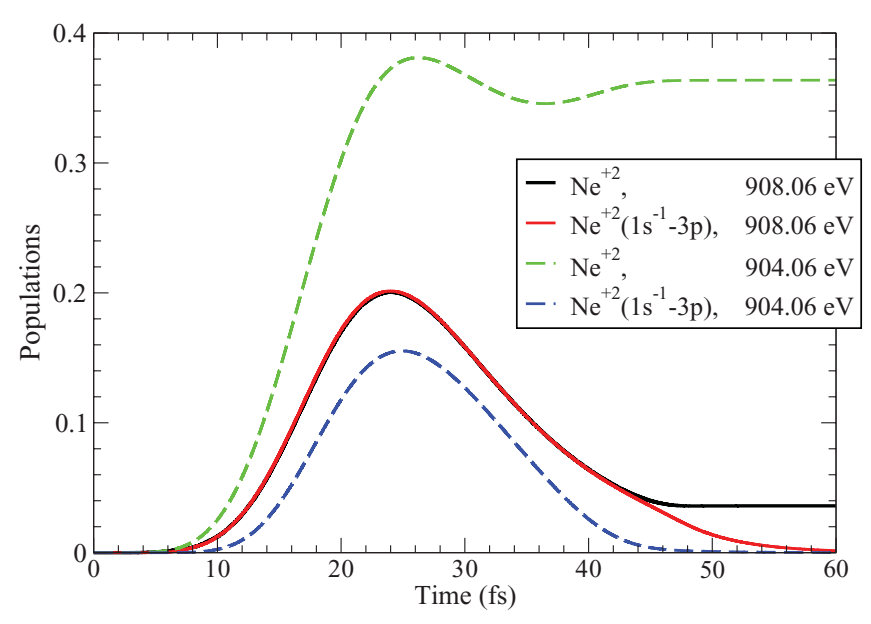

FIG. 4. (Color online) Populations of the $\mathrm{Ne}^{2+}$ and $\mathrm{Ne}^{2+}\left(1 s^{-1}-3 p\right)$ states as a function of time peak intensity $I_{0}=5.0 \times 10^{17} \mathrm{~W} / \mathrm{cm}^{2}$. All other parameters as in Fig. 3 .

\section{CONCLUSION}

We have presented a theory of the Auger kinetic spectra and ionization yields based on the time-dependent density matrix theory which encapsulates all the essential dynamical parameters of the physical processes that are involved such as photoionization, photoexcitation, and Auger transitions. We have examined the AES and the ionic yields in the case where an inner-shell photoionization takes place followed by an Auger decay of the singly charged hole system. We have demonstrated the emergence of ac-Stark splitting of the Auger resonance, resulting from strong Rabi couplings. In addition, ionization yields have been calculated for a range of intensities. We show how to control the branching ratios of various ionic species by varying dynamical parameters of the system, such as Rabi coupling and detunings. The theory was applied to the case of $K$-shell ionization of neon with the photon frequency chosen to match the energy differences between ionic $\mathrm{Ne}^{2+}$ ground and excited states.

In the present study we have put aside the issue of a field undergoing fluctuations suitable for the description of more realistic situations. A more realistic approach is to assume a field with an amplitude undergoing random fluctuations [9]. In general, the fluctuations of the field give rise to a nonzero bandwidth (beyond the Fourier bandwidth) and intensity fluctuations $[23,24]$. The main differences are that the field will excite a number of (nearby) excited states (see, for example, the most important ones in Table I), basically those that lie within the x-ray field bandwidth and that the width of the Auger spectrum will be effectively increased with the x-ray bandwidth. The development of the appropriate theory, capable of describing the field fluctuations, requires a detailed formulation which is beyond the purposes of the present study and it will be the subject of a future work. The essential outcome of the present work; namely, the emergence of the ac-Stark splitting in the Auger spectra under the presence of strong ionic Rabi couplings, will remain and the stochastic nature of the field will mainly affect its observability.

Finally, keeping the focus on the essence of the issue of experimental observability, we refrain from showing the influence of the volume integration in the final results, but its 
precise contribution needs to be evaluated when it comes to the actual experimental conditions.

\section{ACKNOWLEDGMENTS}

One of us (LAAN) gratefully acknowledges discussions with Peter Lambropoulos. A useful comment from
Dr. M. Meyer is appreciated. LAAN is mainly supported by the Science Foundation Ireland (SFI) Stokes Lectureship programme and by the COST Action CM0702. JC acknowledges support from SFI PI 07/IN.1/I1771 and the Higher Education Authority PRTLI IV and V INSPIRE programmes. TJK acknowledges the support of IRCSET.
[1] G. S. Brown, M. H. Chen, B. Crasemann, and G. E. Ice, Phys. Rev. Lett. 45, 1937 (1980).

[2] G. B. Armen, H. Aksela, T. Åberg, and S. Aksela, J. Phys. B: At. Mol. Opt. Phys. 33, R49 (2000).

[3] H. Wabnitz et al., Nature (London) 420, 482 (2002).

[4] H. Wabnitz, A. R. B. deCastro, P. Gurtler, T. Laarmann, W. Laasch, J. Schulz, and T. Moller, Phys. Rev. Lett. 94, 023001 (2011).

[5] W. Ackermann et al., Nat. Photon. 1, 336 (2007).

[6] M. Meyer et al., Phys. Rev. Lett. 104, 213001 (2010).

[7] L. Young et al., Nature (London) 466, 56 (2010).

[8] P. Lambropoulos and P. Zoller, Phys. Rev. A 24, 379 (1981).

[9] N. Rohringer and R. Santra, Phys. Rev. A 77, 053404 (2008).

[10] C. Buth and K. J. Schafer, Phys. Rev. A 80, 033410 (2009).

[11] J.-C. Liu, Y. P. Sun, C. K. Wang, H. Agren, and F. Gelmukhanov, Phys. Rev. A 81, 043412 (2010).

[12] L. S. Cederbaum, Y.-C. Chiang, P. V. Demekhin, and N. Moiseyev, Phys. Rev. Lett. 106, 123001 (2011).
[13] E. P. Kanter et al., Phys. Rev. Lett. 107, 233001 (2011).

[14] S. H. Autler and C. H. Townes, Phys. Rev. 100, 703 (1955).

[15] L. G. Hanson, J. Zhang, and P. Lambropoulos, Phys. Rev. A 55, 2232 (1997).

[16] V. Schmidt, Electron Spectrometry of Atoms Using Synchrotron Radiation (Cambridge University Press, Cambridge, 1997).

[17] P. Pelicon, I. Cadez, M. Zitnik, Z. Smit, S. Dolenc, A. Muhleisen, and R. I. Hall, Phys. Rev. A 62, 022704 (2000).

[18] J. Tulkki et al., Z. Phys. D 5, 241 (1987).

[19] W. H. Louisell, Quantum Statistical Properties of Radiation (John Wiley, New York, 1990).

[20] S. N. Dixit and P. Lambropoulos, Phys. Rev. A 27, 861 (1983).

[21] R. D. Cowan [http://aphysics2.lanl.gov/tempweb].

[22] C. P. Bhalla, N. O. Folland, and M. A. Hein, Phys. Rev. A 8, 649 (1973).

[23] A. T. Georges and P. Lambropoulos, Phys. Rev. A 20, 991 (1979).

[24] P. Zoller, J. Phys. B 15, 2911 (1982). 